multiple objects, displayed dangerous behaviour related to the delusion, and probably suffered from a personality disorder.

This would suggest that dangerousness (related) in male homosexual erotomania, like male heterosexual erotomania, is associated with multiple delusional objects and unrelated dangerous behaviour. Whether these factors are predictive of similar behaviour in female erotomania remains to be seen. In the female homosexual erotomania case quoted (Urbach et al, 1992), there were several delusional objects (possibly up to five) and the individual engaged in both related and unrelated dangerous behaviour.

Doust, J. W. L. \& Christie, K. (1978) The pathology of love: some clinical variants of de Clerambault's syndrome. Social Science and Medicine, 12, 99-106.

Peterson, G. A. \& Davis, D. L. (1985) A case of homosexual erotomania. Journal of Clinical Psychiatry, 46, 448-449.

UrbaCH, J. R., KhallLy, C. \& MrrChell, P. P. (1992) Erotomania in an adolescent: clinical and theoretical considerations. Journal of Adolescence, 15, 231-240.

Regional Psychiatric Centre (Prairies)

RoBin P. D. MENZIES

P.O. Box 9243

Saskatoon

Canada S7K $3 X 5$

\section{Outdated ECT machines}

SIR: Arnott \& Wilkinson (BJP, April 1993, 162, 572-573) found that mean seizure duration and the proportion of seizures lasting longer than $25 \mathrm{sec}$ onds increased when they upgraded their Ectron Duopulse Series 3 ECT (E3) machine to an Ectron Series 5 model (E5). They attributed the increase in successful seizures to the "higher electrical output" of the E5 machine. We encountered similar differences in patients treated in an ECT clinic equipped with a (modified) Series 2 Ectron Duopulse (E2) an earlier version of the E3, identical in terms of stimulus parameters and power output - and one equipped with an E5. We could not confidently attribute the differences to the machine owing to a variety of unmatched variables, for example the clinics catered for different populations (over 65 and under 65 respectively).

Arnott \& Wilkinson did not state whether their machine was the basic (unmodified, E3u) or modified $(\mathrm{E} 3 \mathrm{~m})$ version, and at what setting on the respective machines patients were stimulated. An E3m may, at certain settings, deliver a greater total electrical charge than the E5. The maximum output of the E5 is $400 \mathrm{mQ}$ at $200 \mathrm{ohms}$, compared to $350 \mathrm{mQ}$ for an E2/E3m at the 'ECT2' setting over 6 seconds. The difference in power output between the E5 and E2/E3m (14\%) is, therefore, not great, but they do differ markedly with respect to stimulus intensity. The E5 delivers its maximum power in 3.25 seconds, whereas the E2/E3 does so in 6 seconds. Thus it is only in terms of stimulus intensity that the E5 is 'more powerful' than the E2/E3m.

No-one knows which variable - total electrical charge $(\mathrm{mQ})$, or stimulus intensity $(\mathrm{mQ} / \mathrm{s})$ - is more important (Special Committee on ECT, 1993). A useful, much needed and relatively easy audit research project would be to compare two groups of matched subjects allocated to receive treatment by means of an E5 machine (set at $300 \mathrm{mQ}$ ) or an E3m machine (set at ECT2; stimulus duration 5 seconds).

Special Committee on ECT of the Royal College of PsychiaTRISTS (1989) The Practical Administration of ECT. London: Royal College of Psychiatrists.

\section{Windsor Day Hospital}

HAKeEM KazeEM

\section{Liverpool}

\section{Cognitive function and fall-related fractures}

SIR: Jaborian et al (BJP, July 1994, 165, 122) provide evidence of strong correlation between poor scores on a battery of tests and prior fallrelated fractures in the elderly. They conclude that "low scoring in psychometric tests is a major risk factor for falls".

Correlation is not causation, but a causal link may be the reverse of that suggested, with fat embolism from the fractures impairing cognitive performance.

\section{Jensen Road}

J. D. WILSON

Lincoln LN4 $2 Q U$

\section{Creativity and psychopathology}

SIR: Post's impressive survey of 291 famous men (BJP, July 1994, 165, 22-34) supports a link between high levels of psychopathology and creativity, especially in artists and writers. The psychotic diagnosis for the painter Edvard Munch would further strengthen such a link.

Post lists three artists - Van Gogh, Modigliani and Rossetti-as suffering from a psychotic disorder, all of them organic in nature. My reading of the biographies of Munch is that he suffered from persecutory delusions and auditory hallucinations. Heller (1984) describes Munch's flight from Germany in a vain attempt to avoid his delusional 